\title{
Ultrasonographic Quantification of Local Cardiac Dynamics by Tracking Real Reflectors: Algorithm Development and Experimental Validation
}

\author{
D Adam, A Landesberg, E Konyukhov, P Lysyansky, \\ O Lichtenstein, N Smirin, Z Friedman \\ Technion - Israel Institute of Technology, Haifa, Israel
}

\begin{abstract}
Quantification of cardiac local contraction across the entire ventricle wall is essential for better diagnosis and therapeutic planning. A new ultrasound B-mode image analysis technique was developed that identifies and tracks real reflectors in successive frames. This study tests the ability the technique to identify the mechanical propagation sequence. Cardiac function was monitored (sheep open chest studies, $n=7$ ) by pressure transducers, sonocrystals, and aortic flow meter, concurrent with ultrasound image acquisition. Epicardial electrodes mapped electrical propagation, and allowed different $L V$ pacings. Normal SA node derived activity was compared with abnormal LV contractions. Mechanical indices derived from US images correlated well with the sonocrystals measurements, and the sequence of mechanical contraction with the measured electrical activation. Image analysis allows precise reconstruction of myocardial activation with $16 \mathrm{msec}$ time resolution.
\end{abstract}

\section{Introduction}

Non-invasive quantification of regional myocardial function is one of the main objectives of diagnostic clinical cardiology. Anatomical measures, e.g. myocardial thickening/thinning indices, allow some measure of regional myocardial function. Regional myocardial motion, e.g. local strain/strain rate, are expected to better define regional myocardial function. The various imaging modalities, e.g. 2D echocardiography, angiography, nuclear imaging etc. [1-4], attempt measuring local wall thickening and thinning characteristics, which may better describe regional myocardial function than endocardial motion. Three-dimensional (3D) local wall deformation measurements, in real-time, would be optimal for these purposes, as they can currently be acquired by tagged MRI $[5,6]$. The resolution of Computed Tomography and gated single-photon emission computed tomography/ positron emission tomography (SPECT/PET) imaging enable 3D acquisition of only wall thickening and thinning $[7,8]$. These imaging techniques are not really real-time, their temporal resolution is low, and their availability is low while their price is high. Ultrasound allows real-time acquisition of local thickening/thinning parameters, currently by either grey-scale M-mode recordings (only in 1D) [9], or by Color Doppler myocardial imaging, usually termed Tissue Velocity Imaging (TVI).

TVI, due to its high frame rate format ( $>120$ frames per sec) provides excellent measure of myocardial velocities along the ultrasound image lines $[10,11]$. Although multiple clinical studies have explored the diagnostic role of this technique and have recommended its use, it suffers from two major drawbacks: the amplitude of the estimated velocity depends on the angle at which the region is imaged, thus quantification of the velocities is inaccurate; also, the overall heart motion, translation, rotation and the effects of other myocardial segments destroy the regional velocity estimates [12]. Strain rate imaging, i.e. measuring the rate of deformation, allows estimating spatial gradients of the myocardial velocities and is much less sensitive to artifacts caused by whole heart motion. Local strain can be calculated from the strain rate curves, producing the regional strain imaging.

A novel different approach has been recently developed, based on tracking strong reflectors in 2D Bmode sequences of images. This approach depends on 3 conditions: 1. High rate of image acquisition $(>60$, preferably $>400) ; 2$. Effective filtration of strong anatomical reflectors; 3 . Effective tracking algorithm of such strong reflectors. The present study is based on data obtained using an image system capable of high-rate image acquisition utilizing a frame rate of 60 to $90 \mathrm{fps}$, and a post-processing software that together fulfill all these 3 conditions. The present study is aimed to validate the tracking algorithm, and to evaluate the accuracy of the measured local stains, in controlled open chest animal setting. The study utilized healthy, adult sheep. Different mechanical wave propagation forms (and thus mechanical function modes) were produced by epicardial pacing at different sites, which allowed abnormal electrical propagation at a segment of the activation sequence. 


\section{Methods}

The experiments were performed on 7 sheep, $45-55 \mathrm{~kg}$ in weight, and were conducted according to the guidelines of the Institutional Review Board. Each animal was anaesthetized and the heart was exposed by left thoracotomy. Pressure transducers (Millar) were introduced into the left ventricle and the ascending aorta. Six sonocrystals (Sonometrics) were implanted in the myocardium to measure the left ventricle short and long axis diameters and to reconstruct the global ventricle contraction. Two flow meters (Transonics) were places around the aortic arch and the brachiocephalic artery, to measure the aortic flow and stroke volume. 7 bipolar electrodes (Medtronics) were sutured into the epicardium, along the short axis LV free wall, from the posterior interventricular line to the anterior interventricular line, close to the base. These electrodes allow mapping the propagation of the electrical activity. 2 additional electrodes were sutured at the lateral base and near the anterior septum, and were used to elicit aberrant ventricle excitation. Additional electrode was place on the auricle of the left atrium to identify the atrial activity and to allow appropriate timing of the imposed aberrant ventricular activation with the normal sinus atrial rhythm. Thus, the imposed pacing stimulates the ventricle after the normal SA node activity, and just before the normal excitation through the AV node. Computer controlled software (Labview) allow to define the parameters of the paced activity: delay from the detected SA node activity, duration and amplitude of the ventricular pacing, and the sequence of pacing. Usually an alternate pacing was used, where each normal beat was followed by a paced beat. The measured cardiac function, utilizing sonocrystals and flow meters, allow to compare the US derived indices with well established traditional measurement of global LV function. The measured epicardial electrical activity allows comparing the US derived sequence of mechanical activation with the measured electrical wave propagation. The normal sinus node activity was compared with the paced contractions. ECG, epicardial electrograms, LV and LA pressures and aortic flow and the distances measure by the sonocrystals were all acquired at $1 \mathrm{Khz}$. LV short-axis and long-axis B-mode image sequences were acquired at high rate ( $>60$ FPS) by a commercial US system (GE-Vivid 7 with M5S probe).

Data processing: A new image analysis technique was used that processes cine of frames. The algorithm identifies and tracks real reflectors in successive frames. The algorithm requires initial marking of the mid-wall by the operator, and then selection of a regions-of-interest (ROI). After these manual initial definitions (Fig. 1) the algorithm performs $2 \mathrm{D}$ tissue tracking using the $\mathrm{B}$-mode loops. The algorithm calculates successive 2D correlation coefficients and performs the statistical analysis required to reject random speckles. Since the acquired loops in these experiments included 6-8 cardiac cycles, in which every second beat was LV epicardially paced beat, the mechanical function of the paced beat was compared with the preceding normal beat. The algorithm provides a set of points along the myocardium (Fig. 2). The location and local longitudinal and transverse velocities during the cardiac cycle are calculated and displayed for each point. The 2D tissue tracking procedure was used 3 times in each sequence, to track mostly the endocardial edge, the mid-wall or the epicardial edge, by limiting its ROI to one of these regions at each time. The analyzed images were compared with direct invasive measurements of cardiac contraction and epicardial electrical propagation.

\section{Results}

Each processed sequence of images (either of LV short-axis or long-axis views, 2 heart beats in length) was initially presented in the format of Fig. 1 to an expert, so that the tracking could be evaluated. Then the processing provided the plotting of the longitudinal strain with time, as seen in Fig. 2. In this figure, for clarity, only points along the free wall are depicted.

One of the ways of analysing the data, and evaluating the data produced by the algorithm, is to compare the total strain changes during the cardiac cycle - to other measures that were recorded during the experiment. Since in these experiments the normal beat was followed by a paced one - it allows examining whether unsynchronized and slowed electrical propagation through the LV wall (from the pacing site) which produces noticeable changes in surface ECG and in the electrograms measured by the epicardial leads - also produces noticeable changes in the

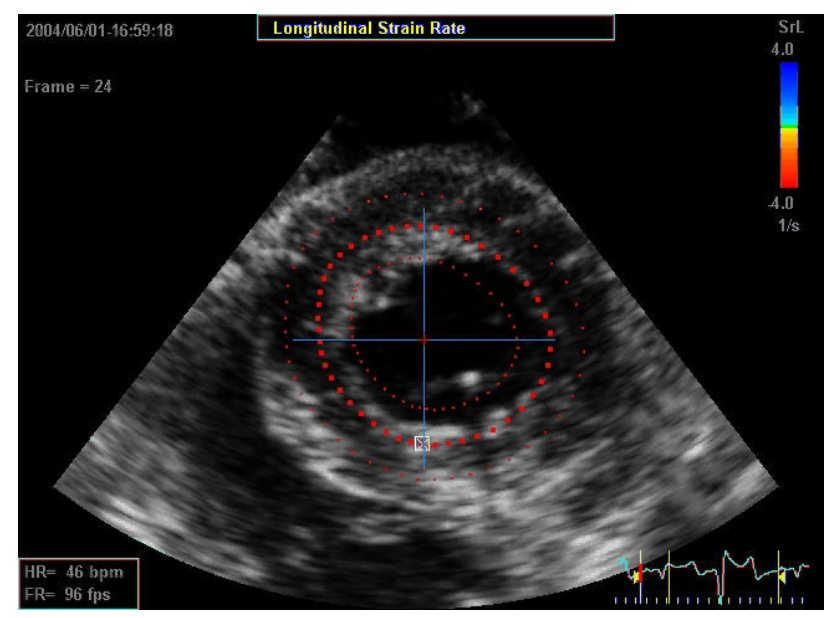

Fig. 1: LV short axis view, with 2 circles of small dotes marking the ROI, and the circle of larger dotes marking the calculated locations, based on tracking the tissue, in which strain and strain rates are calculated. 


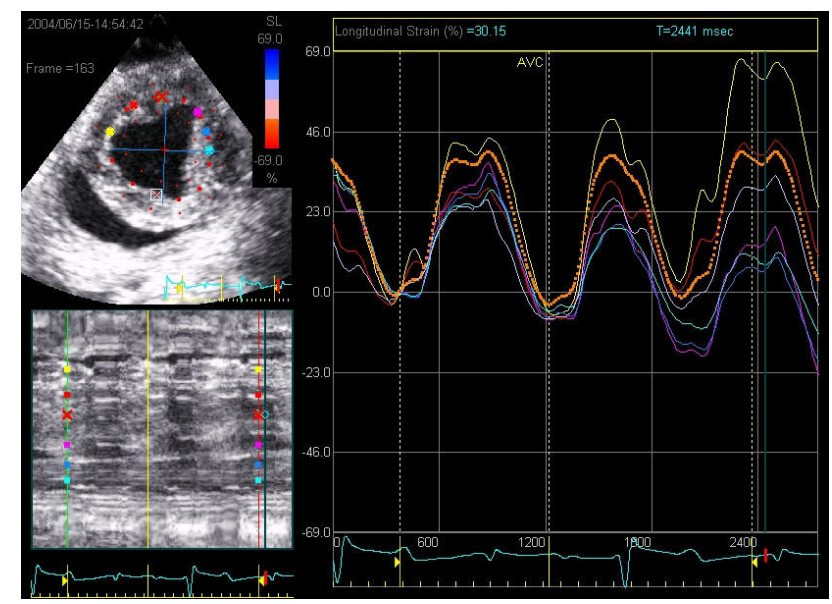

Fig. 2: Display of the longitudinal strain during 3 cardiac cycles, where the $2^{\text {nd }}$ one is a premature beat initiated by LV epicardial septal stimulation.

mechanical order of activation. This examination is highly sensitive, since the difference in the order of activation takes about 50-60 msec, and affects only a small part of the free wall.

Figure 3 presents the measured cardiac mechanical function and epicardial electrical activity during 2 successive normal and paced beats. The different subplots depict (from top to bottom) Aortic \& LV pressures and the time of stimulus by an epicardial lateral LV electrode (a), the aortic flow (b), the epicardial Electrograms (c), and the surface ECG (d). Note the significant change in the ECG introduced by lateral pacing, that inverts the direction of the principle ECG dipole.

To evaluate the global cardiac function based on the tracking of large reflectors, the circumferential strain within the ROI was calculated. Tight correlation was found between the calculated circumferential strain and the short axis diameters measured by the sonocrystals, as shown in Fig. 4. The time course of the circumferential strain resembles the time course of the sonocrystals and tight correlation was found between the estimated fractional changes in the circumferential strain and the fractional shortening in the diameters measured by the sonocrystals.

The novel technique for analysing the US images allows to identify the site of excitation and the direction of the mechanical activation within the US frame rate resolution. Figure 5 presents the strain tracings from 6 different locations along the ventricle wall and the associated surface ECG during normal and paced beat, when the LV was paced by the lateral electrode. While all the six points start to shortening almost instantaneously for the normal electrical excitation (Fig. 5 Left), lateral excitation produced significant dispersion in the (a)

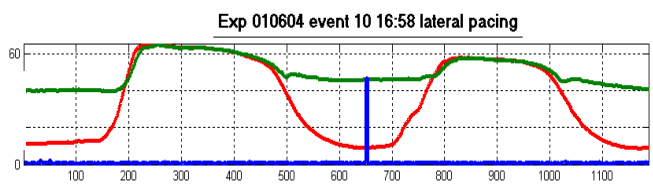

(b)

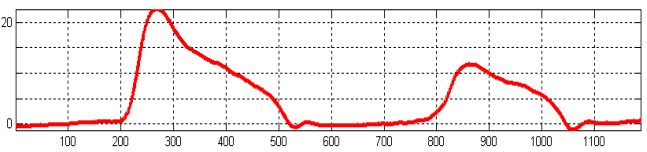

(c)

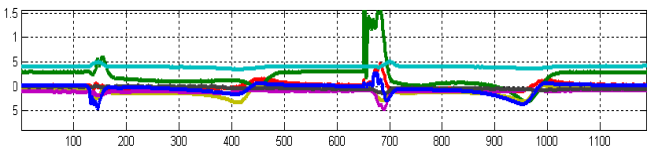

(d)

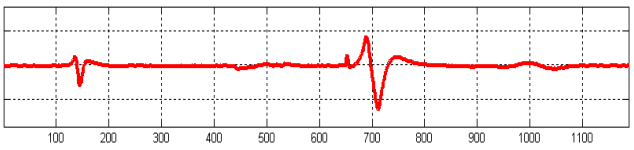

Fig. 3: (a) Aortic \& LV pressures and the time of stimulus by an epicardial lateral LV electrode. (b) Aortic flow. (c) Epicardial Electrograms during the normal and paced beats. (d) ECG demonstrating the PVC effect.

(a)

b)

$\left(c^{\prime}\right.$

(d

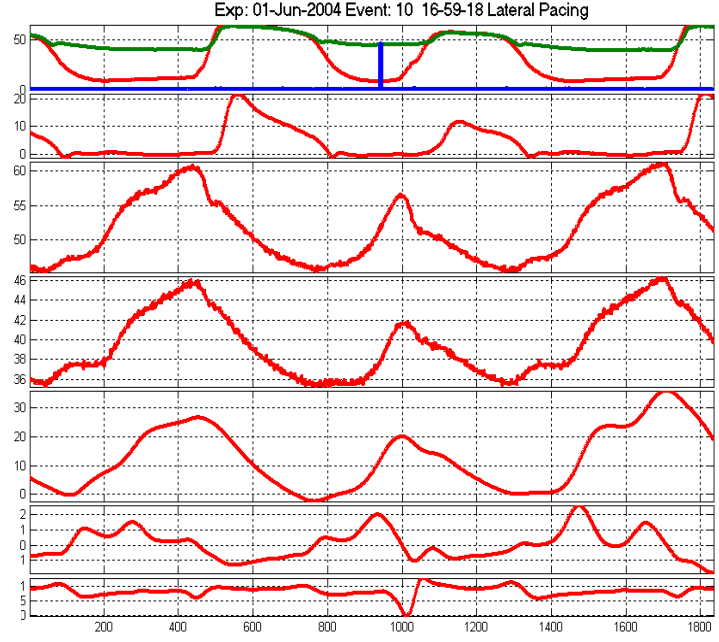

Fig. 4: (a) Aortic \& LV pressures and the time of stimulus by an epicardial lateral LV electrode. (b) Aortic flow. (c) Septal-Posterior distance, and (d) Septal-Lateral distance, both measured by sonocrystals. (e) Circumferential Strain, and (f) Strain-Rate calculated from cine B-mode images. (g) ECG demonstrating the PVC effect.

measured mechanical local strains. Shortening started earlier near the pacing electrode and there was a propagation in the mechanical activation from the lateral to the septal wall. Note also that areas that were activated earlier produce smaller total shorting compared with the 
normal beat. There was a tight correlation between the calculated mechanical activation, as measured from the US images, and the directly measured epicardial electrical activity.

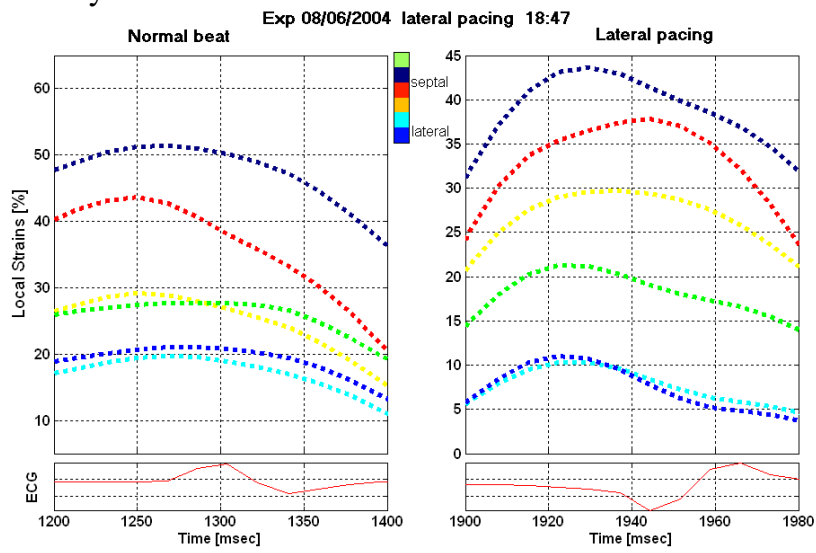

Fig. 5. Strain tracings from 6 different locations along the ventricle wall, and the associated surface ECG during normal (left) and paced beat (right), when the LV was paced by the lateral electrode.

\section{Discussion and conclusions}

A novel technique for processing US images was validated utilizing direct invasive measurements of cardiac contraction and epicardial electrical propagation. Tight correlation was found between the measured US indices, based on tracking large reflectors, and the invasive measurement of cardiac diameters and stroke volume. Moreover, the sequence of the mechanical contraction evaluated from the US images was consistent with the directly measured electrical activation. The image analysis allows precise reconstruction of the myocardial activation with time resolution of $16 \mathrm{msec}$. While regular visual inspection of the US images, without precise detection of the changes and sequence of local strains, allows reasonable evaluation of the cardiac mechanical function, it can not differentiate between reduced local contractility or unsynchronized mechanical activity. Tracking tissue markers allows reconstructing the precise sequence of the mechanical contraction, and the evaluation of the propagation of the mechanical activation wave. This new information is of immense clinical importance, especially in the evaluation of the unsynchronized failing heart.

\section{Acknowledgements}

The authors are grateful for the help and assistance provided by Ms. Levy and Mr. Shenhav. They also acknowledge the support of the Magneton program, the Chief Scientist, Ministry of Industry and Commerce.

\section{References}

[1] Gibson DG, Prewitt TA, Brown DJ. Analysis of left ventricular wall movement during isovolumic relaxation and its relation to coronary artery disease. Br Heart J 1976; 38: $1010-1019$.

[2] Lang RM, Vigon P, Weinert L, Bednarz J, Korcarz C, Sandelski J, Koch R, Prater D, Mor-Avi V. Echocardiographic quantification of regional left ventricular wall motion with color kinesis. Circulation 1996; 93: 1877-1885.

[3] Heger JJ, Weyman AE, Wann LS, Dillon JC, Feigenbaum H. Cross-sectional echocardiography in acute myocardial infarction: detection and localization of regional left ventricular asynergy. Circulation 1979; 60: 531-538.

[4] Cwaijg E, Cwajg J, He ZX, Hwang WS, Keng F, Nagueh SF, Verani MS. Gated myocardial perfusion tomography for the assessment of left ventricular function and volumes: comparison with echocardiography. J Nucl Med 1999; 40: 1857-1865.

[5] Beyar R, Shapiro EP, Graves WL, Rogers WJ, Guier WH, Carey RL, Soulen EA, Zerhouni ML, Weisfeldt ML, Weiss JL. Quantification and validation of left ventricular wall thickening by a three-dimensional volume element magnetic resonance imaging approach. Circulation 1990; 81: 297- 307.

[6] Rademakers FE, Bogaert J. Left ventricular myocardial tagging. Int J Card Imaging 1997; 13: 233-245.

[7] Schermund A, Gerber T, Behrenbeck T, Reed JE, Sheedy PF, Christian TF, Rumberger JA. Measurement of myocardial infarct size by electron beam computed tomography: a comparison with $99 \mathrm{mtc}$ sestamibi. Invest Radiol 1998; 33: 313-321.

[8] Shen MY, Liu YH, Sinusas AJ, Fetterman R, Bruni W, Drozhinin OE, Zaret BL, Wackers FJ. Quantification of regional myocardial wall thickening on electrocardiogramgated spect imaging. J Nucl Cardiol 1999; 6: 583-595.

[9] Guth B, Savage R, White F, Hagan A, Samtoy L, Bloor C. Detection of ischemic wall dysfunction: comparison between m-mode echocardiography and sonomicrometry. Am Heart J 1984; 107: 449-457.

[10] McDicken WM, Sutherland GR, Moran CM, Gordon LN. Colour doppler velocity imaging of the myocardium. Ultrasound Med Biol 1992; 18: 651-654.

[11] Sutherland GR, StewartMJ, Groundstroem WE, Moran CM, Fleming A, Guell-Peris FJ, Riemersma RA, Fenn LN, Fox KAA, McDicken WN. Color doppler myocardial imaging: a new technique for the assessment of myocardial function. J Am Soc Echocardio 1994; 7: 441-458.

[12] Tsutsui H, Uematsu M, Shimizu H, Yamagishi M, Tanaka N, Matsuda H, Miyatake K. Comparative usefulness of myocardial velocity gradient in detecting ischemic myocardium by a dobutamine challenge. J Am Coll Cardiol 1998; 31: 89- 93 .

Address for correspondence

Prof. Dan Adam

Dept. of Biomedical Engineering, Technion, Haifa, Israel

E-mail: dan@biomed.technion.ac.il 